\title{
ECONOMIE Les marchés oléagineux de l'Europe dans le contexte international
}

\section{Economics European oilseed markets in an international context}

Oléagineux, Corps Gras, Lipides. Volume 8, Numéro 3, 183-90, Mai - Juin 2001, La filière

Auteur(s) : Yves DRONNE, Inra, Economie et sociologie rurales, rue Adolphe-Bobierre, 35011 Rennes Cedex.

Résumé : L'Union européenne est un acteur important du marché international des oléagineux. Ses besoins en protéines et en huiles végétales sont extrêmement élevés. Bien que, entre 1980 et 1994, cette zone ait sensiblement augmenté ses surfaces en oléagineux, elle reste un producteur mondial modeste et doit importer de pays tiers l'essentiel de ses besoins en graines, tourteaux et huiles. L'Amérique du Nord et celle du Sud constituent ses principaux fournisseurs, de même que certains pays asiatiques comme la Malaisie ou I'Indonésie, mais les échanges à l'intérieur de l'UE et avec les PECOS se sont nettement développés. Le faible taux de couverture de l'Europe, particulièrement en tourteaux, rend cette zone très vulnérable aux aléas du marché mondial et aux décisions qui peuvent être prises à l'étranger et tout particulièrement aux États-Unis. Alors que la mise en place de I'Agenda 2000, caractérisée par un net recul des surface dès la première année d'application, pose de nouveaux problèmes de compétitivité à ces cultures dans un contexte de faibles prix mondiaux, le maintien de cette filière demeure un bien collectif pour les différents acteurs : consommateurs, agriculteurs, transformateurs et citoyens.

Mots-clés : protéines, tourteaux, UE, PECOS, soja, colza, tournesol.

Summary : EU is an important actor on world oilseeds market. Its needs in vegetable proteins and oils are very high. Though from 1980 to 1994, the area cultivated in oilseeds has notably increased, it remains a marginal producer at world level, and has to import from third-countries most of its needs in seeds, meals and oils. North and South America are its main suppliers, so as, for oils, Malaysia and Indonesia, but trade between EU countries and with CEEC is notably increasing. The low rate of selfsufficiency of Europe, mainly for oilmeals, makes this area very vulnerable to risks on world markets and to decisions taken abroad and particularly in the USA. At a time when the fisrt year of application of Agenda 2000, characterised by a decreases of EU areas in rapeseeds, sunflowerseeds and soybeans, arises new problems of competitivity for these crops in a context of low world prices, the maintenance of this sector is a public good for consumers, crop-producers, crushing and animal feed industries and for citizens.

Keywords : proteins, oilmeals, EU, CEEC, soybean, rapeseed, sunflowerseed. 


\section{ARTICLE}

Les oléagineux constituent un enjeu important pour l'Union européenne (UE). C'est naturellement dans les périodes de crise comme celle liée aux farines de viande, environ 25 ans après celle qu'avait constitué l'embargo sur les exportations de soja des États-Unis, que ces enjeux sont les plus apparents. Ces à-coups traduisent en fait un problème de fond, qui ne peut recevoir que des solutions de continuité. L'UE est un géant sur la scène mondiale en termes de consommation de tourteaux et d'huiles, de même qu'en termes de trituration, mais c'est un géant aux pieds d'argile car ces utilisations reposent sur une production locale de graines qui reste modeste et vulnérable dans la nouvelle phase de turbulence que constitue l'application de l'Agenda 2000.

Les marchés européens des oléagineux et de leurs co-produits (huiles et tourteaux) sont complexes puisque, avec des récoltes de graines réparties dans six principaux pays - même si la France continue à jouer un rôle majeur -, ils sont constitués de flux d'échange importants de graines, de tourteaux, d'huiles brutes et d'huiles raffinées entre les divers pays membres, mais aussi, de plus en plus, avec les pays d'Europe centrale et orientale (PECOS) et certains autres pays du continent comme l'Ukraine et la Russie. Dans ce système d'interdépendance qui s'est mis en place au cours des vingt dernières années, chaque pays présente certaines spécificités en termes de production et de transformation de graines, ainsi que de consommation d'huiles et de tourteaux. Cette hétérogénéité est à la fois le reflet des différences dans les conditions climatiques et agronomiques des pays, dans les habitudes alimentaires des consommateurs pour les huiles et dans les systèmes d'élevage pour les produits animaux.

Bien que caractérisé par un très fort déficit en graines, en tourteaux et en huiles, un régime de croisière, plus ou moins "satisfaisant ", s'était établi grâce aux efforts importants des agriculteurs pour diversifier leurs productions et mieux répondre aux signaux de la demande intérieure et des marchés mondiaux -, des industriels - pour moderniser et concentrer leur secteur, demeurant ainsi compétitifs au niveau mondial -, de la recherche - pour améliorer la qualité et les rendements de ces graines -, et enfin des pouvoirs publics - pour, durant toute une période, encourager l'expansion de ces cultures.

Dans cet article, on abordera successivement quatre points. D'abord, on rappellera la place de l'UE dans le monde en termes de production, de commerce et de consommation, et on caractérisera la structure actuelle de la filière UE des oléagineux par rapport aux autres zones. Ensuite, on analysera certaines grandes évolutions de la situation communautaire et on les resituera dans les grands mouvements qui affectent le contexte international.

\section{Quelques chiffres sur I'UE dans le monde}

Sur le marché mondial des oléagineux, l'UE occupe des places très différentes selon les niveaux de cette filière qui va de la production de graines à la consommation d'huiles et de tourteaux. Ces différences peuvent être illustrées à travers le rappel de quelques chiffres et pourcentages concernant la campagne 1999/2000.

* Avec $45 \%$ du total mondial, l'UE est de loin le premier importateur de tourteaux ${ }^{2}$ devant la Corée du Sud (2,2 Mt), la Chine (1,5 Mt) et la Thaïlande (1,4 Mt). Sur un total mondial de 51 millions de tonnes (Mt), I'UE représente à elle seule $23 \mathrm{Mt}$ et précède largement l'ensemble des PECOS qui 
arrivent en seconde position avec près de $3 \mathrm{Mt}$. Pour le seul tourteau de soja, les importations de I'UE s'élèvent à $17 \mathrm{Mt}$ (soit $74 \%$ du total des importations de tourteaux), chiffre qui est voisin de la part de ce tourteau dans le commerce mondial (73\%). L'UE est donc à la fois le premier importateur mondial de tourteau de soja (loin devant tous les autres pays et en particulier les PECOS qui arrivent au second rang avec à peu près $2,8 \mathrm{Mt}$ ) et un importateur majeur de certains autres tourteaux. Ainsi I'UE est le premier importateur mondial de tourteau de tournesol (1,8 Mt sur 2,5 soit $72 \%)$, le premier importateur de tourteaux de coprah, d'arachide, de lin et de palmiste, la Corée arrivant souvent en deuxième position mais loin derrière.

* Avec 32 \%, l'UE est le premier importateur mondial de graines oléagineuses (surtout de soja, mais aussi colza et tournesol). Avec plus de $20 \mathrm{Mt}$ sur un total mondial de $63 \mathrm{Mt}$ pour l'ensemble des graines, I'UE conserve de loin la première position devant la Chine (11 Mt), le Japon (7,6 Mt) ou le Mexique (4,9 Mt). Pour le seul soja ${ }^{3}$, I'UE, avec plus de $16 \mathrm{Mt}$ (soit $33 \%$ du commerce mondial), garde la première position devant la Chine $(10 \mathrm{Mt})$, le Mexique et le Japon qui ont des tonnages compris entre 4 et $5 \mathrm{Mt}$. Le soja représente nettement la première graine importée dans I'UE avec 80 $\%$ du total, mais I'UE est aussi, et de loin, le premier importateur mondial de graines de tournesol avec plus de $2 \mathrm{Mt}$ sur un total de $3 \mathrm{Mt}$.

* Avec 25 \%, l'UE est le premier consommateur mondial de tourteaux loin devant les États-Unis. Selon les dernières estimations d'Oil World, I'UE devrait consommer au cours de la campagne 2000/2001 environ $43 \mathrm{Mt}$ de ces produits sur un total mondial de $182 \mathrm{Mt}$, ce qui la place nettement devant les États-Unis et la Chine avec chacun environ $32 \mathrm{Mt}$. Pour le seul tourteau de soja qui représente $28 \mathrm{Mt}^{4}$, I'UE arrive en première position à égalité avec les États-Unis. Ces deux pays représentent encore, à eux seuls, plus de la moitié de la consommation mondiale de tourteau de soja.

* Avec $15 \%$, l'UE est le deuxième consommateur mondial de corps gras végétaux (13 Mt sur un total de $91 \mathrm{Mt}$ ), derrière la Chine (13 Mt), mais devant les États-Unis et I'Inde (10 Mt chacun). L'UE consomme en outre de l'ordre de 4,7 Mt d'huiles et graisses animales, surtout sous forme de beurre, de suif et de lard. Même si I'huile d'olive conserve une importance considérable dans les utilisations alimentaires avec plus de 1,6 Mt, les huiles de colza, de soja, de tournesol et même de palme représentent aujourd'hui des tonnages sensiblement plus élevés. Selon les produits, les utilisations non alimentaires de ces produits sont plus ou moins fortes.

* Avec $13 \%$, l'UE est le deuxième importateur mondial de corps gras végétaux (4 Mt sur un total mondial de $32 \mathrm{Mt}$ ), surtout sous forme d'huile de palme et d'autres huiles tropicales, derrière I'Inde (près de $6 \mathrm{Mt}$ ), mais devant la Chine (2,5 Mt). De plus, I'UE importe environ 450000 tonnes de corps gras animaux. L'UE est par ailleurs, avec $1,8 \mathrm{Mt}$ à destination des pays tiers, le quatrième exportateur mondial de corps gras (surtout d'huiles de colza et de soja) loin derrière la Malaisie, I'Indonésie et l'Argentine (qui dépassent largement ou atteignent les $5 \mathrm{Mt}$ ), mais devant les États-Unis et le Brésil.

* Avec 12 \%, l'UE est le troisième triturateur mondial (de graines communautaires et importées). Ce pourcentage correspond à $31 \mathrm{Mt}$ sur un total mondial de $260 \mathrm{Mt}$. L'UE arrive après les États-Unis (50 Mt dont $41 \mathrm{Mt}$ en soja) et la Chine (44 Mt dont $18 \mathrm{Mt}$ en soja), mais devant le Brésil (24 Mt), l'Argentine (23 Mt) et l'Inde (19 Mt). Pour le seul soja, la transformation de l'UE, avec $16 \mathrm{Mt}$ sur 145 Mt dans le monde, correspond à $11 \%$. 
* Avec seulement 5 \%, l'UE est, malgré son tonnage relativement limité (environ 14 Mt en 2000/2001 contre près de 17 l'année précédente), le sixième producteur mondial d'oléagineux. Sa récolte, à rapprocher d'un total mondial de $298 \mathrm{Mt}$, la situe très loin après les États-Unis (87 Mt), la Chine (43 Mt), le Brésil (36 Mt), l'Argentine (29 Mt) et l'Inde (22 Mt). Alors qu'au niveau mondial le soja représente $56 \%$ de la production de graines, il ne constitue que $8 \%$ de celle de l'UE qui comprend pour l'essentiel des graines de colza et de tournesol.

\section{Représentation graphique du système oléagineux}

Les pourcentages indiqués précédemment qui vont de 45 à $5 \%$ traduisent le fait que pour l'UE, comme pour de nombreux autres pays globalement déficitaires ou excédentaires, il existe des différences très fortes dans le poids de la production de graines, de la transformation et de la consommation. Le fait qu'il existe deux débouchés simultanés pour les graines - qui sont d'une part I'huile et d'autre part le tourteau, chacun de ces co-produits ayant une importance plus ou moins grande selon la nature de la graine - pose un véritable problème de représentation de l'équilibre de chaque pays. Dans ce texte, nous avons privilégié l'aspect " tourteau ». Cependant, la même représentation peut s'appliquer aux huiles végétales, même si certaines d'entre elles ne proviennent pas de graines oléagineuses (olive, palme, etc.).

De façon générale, la structure du marché des oléagineux et des tourteaux à l'intérieur d'une zone géographique donnée peut se représenter sous forme d'une pyramide tronquée. La largeur de la base de cette pyramide correspond à la production de graines exprimée en équivalent tourteaux. La largeur de la pyramide à mi-hauteur correspond à la production totale de tourteaux (à partir de graines locales et importées). Enfin, la largeur du « sommet » de la pyramide plus où moins tronquée correspond à la consommation de tourteaux. Par différence, l'écart entre la base et la largeur à mihauteur correspond au solde en graines oléagineuses exprimé en équivalent tourteaux et l'écart entre la largeur à mi-hauteur et celle du " sommet » correspond au solde en tourteaux. La différence totale entre la base et le sommet représente, exprimé en équivalent tourteaux, le déficit ou l'excédent global de la zone. De plus, les rapports entre la base et le sommet et entre la largeur à mihauteur et le sommet correspondent au taux de couverture respectivement primaire et secondaire. Plus ces taux de couverture sont élevés plus les utilisateurs (triturateurs ou fabricants d'aliments composés) ont de facilité à s'approvisionner sur le marché local et donc de probabilité de bénéficier d'une bonne compétitivité.

Ainsi, pour les États-Unis (figure 1), la pyramide repose sur une base extrêmement large (64 Mt d'équivalent tourteaux). Elle a une forte largeur à mi-hauteur (38 Mt de tourteaux) et un " sommet » un peu plus étroit ( $32 \mathrm{Mt}$ ). On voit que cette pyramide présente un fort rétrécissement entre la base et la largeur à mi-hauteur, puis une partie supérieure presque verticale. Cela illustre le fait que les États-Unis sont principalement des exportateurs de graines (pour l'équivalent de $26 \mathrm{Mt}$ ) et beaucoup plus modestement des exportateurs de tourteaux (pour environ $6 \mathrm{Mt}$ ).

Par rapport aux États-Unis, le Brésil et l'Argentine (figure 2) ont tous deux des pyramides qui sont appuyées sur de larges bases, respectivement 26 et $19 \mathrm{Mt}$, mais qui présentent un fort renflement à mi-hauteur (niveau de la trituration) suivi d'un très fort rétrécissement et d'un " sommet » relativement étroit pour le Brésil et très " pointu » pour l'Argentine. Ce dernier pays, en raison de sa faible population et de sa faible production de viandes blanches, reste un « petit » consommateur de tourteaux avec moins d'un million de tonnes, ce qui contraste avec son poids considérable sur le 
marché mondial des tourteaux.

Pour l'UE (figure 1), on a une grande pyramide (qui est certes un peu moins importante que celle des États-Unis) qui présente la caractéristique d'être totalement inversée. Elle repose sur une base étroite de $9 \mathrm{Mt}$ d'équivalent tourteaux, s'élargit à mi-hauteur $(22 \mathrm{Mt})$ et encore plus jusqu'au sommet dont la largeur correspond à $43 \mathrm{Mt}$. Ceci montre bien l'importance du déficit de l'UE, d'une part, en graines (13 Mt d'équivalent tourteaux) et, d'autre part, en tourteaux (21 Mt). Le taux de couverture primaire est égal à $21 \%$ et le taux de couverture secondaire à $51 \%$.

La forme générale de la pyramide représentative de l'UE traduit bien l'instabilité de sa structure d'approvisionnement en graines et tourteaux et sa forte vulnérabilité aux aléas du marché mondial. À l'intérieur de l'UE, pour les différents pays membres, on retrouve dans tous les cas des structures de pyramides inversées qui caractérisent des situations globalement déficitaires. Cependant, on peut distinguer deux formes particulières (figure 3) :

- celle d'une pyramide avec un resserrement au milieu : c'est le cas de la France qui a une situation d'exportateur net de graines (ses exportations de colza et tournesol faisant beaucoup plus que compenser ses importations de soja), de faible triturateur et de gros consommateur de tourteaux, ceux-ci étant pour une large part importés en l'état de pays tiers ou du reste de l'UE ;

- celle d'une pyramide avec une base notable et un premier étage important voisin de la largeur du sommet. C'est le cas de l'Alle-magne, qui a à la fois une base agricole notable, une forte trituration fondée sur beaucoup d'importations de graines, et une consommation de tourteaux qui est globalement faiblement déficitaire.

Par ailleurs, certaines pyramides sont spécifiques dans la mesure où elles ont une base très étroite, voire pratiquement nulle comme aux Pays-Bas. Ces pyramides sont plus ou moins évasées à mihauteur et au " sommet " selon que l'approvisionnement du pays s'effectue surtout sous forme de graines ou surtout sous forme de tourteaux.

Concernant ce type de représentation, on doit noter deux points importants. Tout d'abord, le fait pour un pays d'avoir une largeur à mi-hauteur (c'est-à-dire une production de tourteaux) voisine de celle du sommet (c'est-à-dire de la consommation) ne préjuge en rien de l'importance du commerce extérieur en raison des échanges croisés qui existent souvent pour différents tourteaux. Ainsi, I'Allemagne, qui a un déficit global en tourteaux de 0,8 Mt, est à la fois un gros exportateur net de tourteau de colza et aussi un important exportateur et importateur de tourteau de soja (avec un solde négatif pour ce produit d'environ $1 \mathrm{Mt}$ ). Une partie de ces échanges s'effectue à l'intérieur de I'UE et avec les PECOS et traduit les interdépendances qui existent de plus en plus entre pays européens.

En second lieu, ces diagrammes correspondent à une photographie de la situation d'un pays ou d'une zone géographique à un instant donné. Or, chacune des trois composantes de la pyramide a évolué dans le passé et continuera d'évoluer, en fonction de paramètres qui lui sont $a$ priori spécifiques ${ }^{5}$ :

- le niveau de la production de graines oléagineuses dépend des rendements moyens de ces cultures, mais en premier lieu des surfaces ensemencées qui, depuis 1993, dépendent des taux de jachère obligatoires annuels, mais surtout de la compétitivité des oléagineux par rapport aux autres cultures. Cette compétitivité fait intervenir à la fois les prix reçus par les agriculteurs (qui, pour les oléagineux, 
découlent des prix mondiaux après conversion par les taux dollar/euro) et des éléments réglementaires, à commencer par les niveaux des aides directes versées aux différentes cultures dans les différentes régions ;

- le niveau de la production de tourteaux dépend pour sa part de l'évolution des capacités de trituration totale de chaque zone (avec éventuellement ouverture ou fermeture de certaines usines), des taux d'utilisation de ces capacités et du choix des graines triturées. Celui-ci dépend en particulier de l'évolution des différentiels de marges de trituration entre graines (colza, tournesol, soja), mais aussi de certaines rigidités au niveau de la demande (tourteau de soja pour l'alimentation animale, huile de tournesol pour la consommation humaine, huile de colza pour le bio-diésel, etc.) ;

- la demande de tourteaux dépend, quant à elle, surtout du niveau de développement des productions animales (en particulier de viandes blanches) et, dans une certaine mesure, de lait et d'œufs, qui se répercute fortement sur la consommation et la production d'aliments composés et donc, in fine, sur la demande des différents ingrédients. La demande de tourteaux dépend alors des rapports de prix avec les céréales, mais aussi des spécificités des animaux produits (porcs, volailles standard, volailles label, produits biologiques, etc.) qui nécessitent des aliments ayant des concentrations énergétiques et protéiques différentes de leurs aliments et peuvent être soumis à certains cahiers des charges qui restreignent les possibilités de substitution entre matières premières.

\section{Grandes évolutions des marchés de l'UE}

Pour chacun des trois grands postes du bilan (production de graines, trituration, consommation de tourteaux), les évolutions ont été fortes au cours des dernières années.

\section{Production de graines}

Pour les 15 pays actuels de l'UE, les surfaces en oléagineux (figure 4), après une longue stagnation consécutive à la mise en place de l'Organisation commune de marché (OCM) des " matières grasses » en 1966, ont connu une période de forte croissance entre 1979 et 1987, avec une augmentation de 3,5 millions d'hectares (soit une progression moyenne de plus de 500000 ha/an). Avec la mise en place de quantités maximum garanties $(\mathrm{QMG})^{6}$, puis la réforme du règlement oléagineux dès 1992/1993, son intégration dans le cadre de la réforme Mac Sharry de la PAC en 1993/1994, I'introduction en 1994/1995 des surfaces maximum garanties (SMG) en application des accords de Blair House ${ }^{7}$, les surfaces n'ont ensuite augmenté qu'à un rythme beaucoup plus lent pour attendre 6,1 millions d'hectares en 1999 contre 5,1 en 1987. Encore cette progression des surfaces s'expliquet-elle largement par le développement des cultures non alimentaires sur jachère (particulièrement de colza) qui, introduites en 1993/1994, ont atteint 1,14 million d'hectares en 1999 . Au cours de cette année, l'UE a été proche de la saturation de l'équivalent million de tonnes de tourteau de soja fixé par Blair House.

En fait, les phénomènes les plus notables sont l'absence de développement important des surfaces en soja aussi bien en France qu'en Italie (on avait pourtant atteint plus de 400000 ha dans ce pays en 1991) et la chute importante des surfaces en tournesol au cours des dernières années (surtout en Espagne, en France et en Italie), recul qui a été, jusqu'à l'application de l'Agenda 2000, compensé par une progression des surfaces en colza alimentaire et non alimentaire, essentiellement en France, en 
Allemagne et au Royaume-Uni. La première année d'application de l'Agenda 2000 se caractérise par de fortes diminutions des surfaces, surtout en colza (- 500000 hectares pour l'UE) dans presque tous les pays. Avec plus de 100000 ha de baisse, le recul concerne surtout le Royaume-Uni, la France et I'Allemagne. Les surfaces en tournesol reculent également (- 150000 ha pour I'UE), notamment en Espagne et en France.

Les rendements constituent, avec les prix et les aides, un élément essentiel de la compétitivité des oléagineux. Pour l'ensemble de l'UE, les niveaux atteints pour le colza, le soja et, dans une certaine mesure, le tournesol ont eu tendance à sensiblement augmenter au cours des quinze dernières années (figure 5) ; cependant, lorsque l'on rapporte ces évolutions de rendements à celle des céréales (figure 6), on constate globalement un phénomène de stagnation, voire de diminution des rendements relatifs des oléagineux. Des espoirs importants de nouveaux progrès de rendement existent pour les oléagineux (notamment pour le colza) qui n'ont pas bénéficié de la même antériorité dans la sélection que les céréales, pourvu que ces cultures continuent à bénéficier d'un effort de recherche important public ou privé.

\section{Trituration}

Le tonnage de graines oléagineuses triturées dans les 15 pays actuels de I'UE a connu une croissance presque ininterrompue au cours des trente dernières années (figure 7) avec une progression moyenne de près de 850000 tonnes par an. Cependant, le phénomène le plus important est la relative stabilisation depuis plus de vingt ans des transformations de soja. Dès 1978, les 15 pays de I'UE transformaient 15,5 Mt de soja, soit presque autant qu'aujourd'hui. Pour les autres oléagineux surtout d'importation (arachide, coton, lin, coprah, palmiste, etc.), le tonnage total qui demeure limité n'a pratiquement pas bougé sur cette période. Ce sont donc les deux principales graines " communautaires » (le colza et le tournesol) qui ont assuré, presque à elles seules, l'augmentation de l'activité de l'industrie de la trituration. Au départ, cette expansion s'est appuyée à la fois sur des importations (notamment du Canada et d'Argentine) et sur l'accroissement des récoltes communautaires. Aujourd'hui, cette activité est essentiellement liée à la production européenne, même si certaines importations subsistent. La place de l'Argentine, qui triture de plus en plus de tournesol sur place et s'est fortement reportée sur le soja a fortement baissé dans ces importations au profit des pays de l'Est de l'Europe ; le Canada, qui s'est fortement orienté dans la production de colza OGM, a pratiquement disparu de la liste des fournisseurs de I'UE au profit des PECOS. Le changement de réglementation appliqué au secteur oléagineux à partir de 1992/1993 ${ }^{8}$ s'est traduit par une plus grande dépendance par rapport à la conjoncture mondiale et s'est accompagné d'une modernisation et d'une concentration du secteur de l'huilerie.

\section{Consommation de tourteaux}

La consommation de tourteaux dans I'UE (figure 8), très faible à la fin des années 60, n'a pratiquement pas cessé de croître depuis, même si cette croissance s'effectue depuis 1985 à un rythme plus lent. On est passé durant cette période de $31 \mathrm{Mt}$ à $40 \mathrm{Mt}$ en 1999. Cela correspond à une progression moyenne annuelle de 650000 tonnes contre un rythme antérieur nettement supérieur au million de tonnes. Malgré les progressions importantes des utilisations de tourteaux de colza et de tournesol, le soja a continué a augmenter fortement (+ $6 \mathrm{Mt}$ depuis 1985). Toutefois, le phénomène important à noter est l'évolution de la place du soja et des autres tourteaux (figure 9). Après une forte progression jusqu'en 1981 où la part du tourteau de soja atteignait 74 \%, celle-ci a 
ensuite reculé jusqu'à 61 \% en 1996, avant de connaître une certaine reprise au cours des trois dernières années. La consommation des " autres tourteaux " (lin, coton, coprah, palmiste), restée à peu près constante en tonnage, a fortement reculé en pourcentage de $32 \%$ au milieu des années 60 à moins de $11 \%$ aujourd'hui. Au niveau de l'ensemble des matières riches en protéines, la place des tourteaux (principalement ceux de soja, colza et tournesol), qui était déjà très forte, est appelée à se renforcer avec la disparition, provisoire ou définitive, des farines de viande et le renchérissement des farines de poissons. Les protéagineux, à commencer par le pois mais aussi la féverole, constituent également des sources de protéines concentrées intéressantes, mais la disponibilité de ces produits sur le marché européen reste limitée.

\section{Consommation d'huiles et corps gras}

Les huiles constituent l'autre co-produit des graines oléagineuses et, dans le cas du colza et du tournesol, celui qui, dans la plupart des conjonctures mondiales, assure la plus grande partie de la valorisation des graines. En termes d'utilisation totale (alimentaire et non alimentaire), sur 17,6 Mt de corps gras enregistrés en 1999/2000, les huiles de graine représentent plus de 7,6 Mt contre 1,7 pour l'olive et 3,4 pour les huiles tropicales, le solde correspondant aux corps gras animaux. Ces chiffres correspondent à un niveau moyen de consommation par tête de $47 \mathrm{~kg} / \mathrm{an}$ qui représente environ 2,5 fois la moyenne mondiale (18 kg/tête/an). Ce chiffre a eu tendance à augmenter dans I'UE au cours des dix dernières années, mais cela est surtout dû au non-alimentaire, la consommation en huile des ménages étant pour sa part à peu près stagnante. L'UE présente un solde déficitaire en corps gras d'environ $2 \mathrm{Mt}$, mais se trouve à la fois très forte importatrice d'huiles tropicales (palme, coprah, palmiste) et forte exportatrice d'huiles de colza, et surtout d'huiles de soja issues pour l'essentiel de la transformation de graines importées. En huile de tournesol, le solde de l'UE est à peu près équilibré.

\section{Échanges intra-communautaires}

En fait, on ne peut considérer l'UE comme un ensemble homogène. Alors que la production de graines est surtout localisée en France, en Allemagne et au Royaume-Uni (tableau), la trituration se trouve surtout en Allemagne, aux Pays-Bas et en Espagne, la consommation de tourteaux principalement en France, en Allemagne, en Espagne et aux Pays-Bas, et celle d'huiles végétales en Allemagne et au Royaume-Uni.

Ces différences de localisation conduisent à des flux de produits importants à l'intérieur de l'UE. Ainsi, en 1999, les échanges intra-communautaires de graines ont porté sur 4,3 Mt (alors que les importations en provenance des pays tiers représentaient plus de $20 \mathrm{Mt}$ ), avec pour l'essentiel du colza et du tournesol (mais aussi plus d'1 Mt de réexportations de graines de soja de pays tiers). La France est de loin le premier fournisseur de ce marché devant le Royaume-Uni et l'Allemagne, tandis que ce dernier pays avec les Pays-Bas et la Belgique constituent le principal débouché. En huiles végétales le commerce intra-communautaire a porté sur 3,3 $\mathrm{Mt}$ (surtout du colza, du tournesol, et du soja, mais aussi des réexportations d'huile de palme) contre 3,5 Mt pour les importations en provenance des pays tiers. Pays-Bas, Allemagne et Belgique sont les principaux fournisseurs à destination de la France, de l'Italie et du Royaume-Uni. II existe de nombreux flux croisés entre certains pays (comme la France et l'Allemagne) d'exportation d'une huile sous forme brute et d'importation de la même huile ou de certaines autres sous forme raffinée. En tourteaux, le commerce intra-communautaire a représenté 6,1 Mt en 1999 (contre des importations en 
provenance de pays tiers de plus de $22 \mathrm{Mt}$ ). Les Pays-Bas, l'Allemagne et la Belgique sont les principaux fournisseurs, alors que la France, suivie du Royaume-Uni, de la Suède et du Danemark sont les principaux débouchés

\section{Échanges avec les PECOS}

Durant les dernières années, les échanges entre I'UE et les PECOS se sont sensiblement développés. En 1998, sur les 2,6 Mt de tourteaux importés par ces derniers (principalement par la Pologne et les Républiques tchèque et slovaque) 1,1 Mt venait des pays européens contre seulement $0,9 \mathrm{Mt} \mathrm{du}$ Brésil et 0,3 de l'Argentine, les importations en provenance des États-Unis étant de seulement 0,1 Mt. Les PECOS constituent la principale destination des exportations de I'UE qui sont toutefois limitées à environ $5 \%$ de la production locale.

De plus, en 1999/2000, l'UE, probablement au détriment d'un approvisionnement local satisfaisant en tourteaux et huiles de certains des PECOS, a importé 750000 tonnes de graines de tournesol de ces pays (principalement de Roumanie, Bulgarie et Hongrie), environ $1 \mathrm{Mt}$ étant par ailleurs importé de Russie et d'Ukraine. Ceci correspond à une claire réorientation des circuits d'approvisionnement de l'Europe de l'Ouest des pays d'Amérique du Nord (États-Unis) et du Sud (Argentine) vers les pays de l'Est de l'Europe.

Pour le colza, environ 650000 tonnes ont été importées par l'UE durant la même année en provenance des PECOS (principalement des Républiques tchèque et slovaque, de Hongrie et de Pologne). Pour le soja, la Serbie/ Montenegro (320 000 t), la Roumanie (120 000 t) et la Croatie (110 000 t) sont les principaux producteurs de la région en 1999/2000. La production totale des PECOS avec environ 650000 tonnes, ne représente actuellement que la moitié de celle de I'UE, mais a connu au cours des cinq dernières années un notable développement tandis que celle de I'UE stagnait. La région est malgré tout largement déficitaire pour cette graine.

Alors que, pour les céréales, I'UE présente un net excédent (figure 10) et les PECOS de fortes fluctuations du solde du commerce extérieur avec une tendance positive (figure 11), ces zones sont toutes deux largement déficitaires en tourteaux. Pour les graines oléagineuses, alors que le déficit de I'UE n'a cessé de croître au cours des trente dernières années, le solde global des PECOS est actuellement équilibré, voire légèrement excédentaire, les exportations de tournesol et de colza faisant plus que compenser leurs importations de graines de soja. Cette situation globale traduit en fait un certain rationnement de la demande intérieure qui pourrait être beaucoup mieux satisfaite par un développement de ces cultures.

\section{Quelques évolutions de la structure au niveau mondial}

Les évolutions de la filière oléagineuse de I'UE ont été réalisées sous la contrainte d'accords internationaux qui, depuis l'origine de la PAC, prévoient une totale ouverture sur les marchés mondiaux. Durant les périodes, fastes pour les consommateurs et les éleveurs, où les prix internationaux des huiles et des tourteaux sont faibles et l'offre mondiale abondante, la fragilité de la situation communautaire peut être masquée, mais rien n'assure que de telles situations soient appelées à se poursuivre durablement compte tenu de la croissance démographique et économique dans de nombreux pays du monde. Les États-Unis, le Brésil ou l'Argentine ne produisent pas du soja parce que I'UE en a besoin mais parce que, à un moment donné, compte tenu de la situation des 
marché mondiaux et des politiques agricoles mises en place dans ces différents pays, cette culture est, pour les agriculteurs, plus rentable que d'autres. On a connu au cours des dernières années une forte progression de l'offre de soja - et aussi de palme - sur le marché mondial, mais la situation peut évoluer très rapidement, de même que la place de l'Europe sur ce marché. L'UE, qui ne reçoit plus que $22 \%$ des exportations de graines et $8 \%$ des exportations de tourteaux de soja des États-Unis, n'est plus le point de repère des États-Unis qui ont en grande partie laissé ce marché aux pays d'Amérique du Sud. Face au ralentissement de la demande européenne, le plus fort dynamisme de la demande mondiale se trouve aujourd'hui, pour les tourteaux et les graines, en Asie et en Amérique du Sud et, pour les huiles, dans ces deux zones ainsi qu'en Afrique, la Chine constituant bien sûr, à elle seule, un enjeu primordial.

On peut caractériser l'évolution du contexte mondial au cours des dix dernières années par quelques éléments principaux.

Alors que la superficie des céréales restait pratiquement stable aux environs de 700 millions d'hectares, celle des oléagineux a dans le même temps progressé de 35 millions d'hectares (figure 12) soit plus de $21 \%$, tandis que la production augmentait de 84 millions de tonnes (+ $39 \%)$. À l'augmentation des superficies s'ajoute donc, pour les oléagineux, un effet rendement très net qui tient en partie au fait que, dans la plupart des pays et pour les diverses graines, les rendements unitaires ont eu tendance à augmenter mais, aussi et surtout, au fait que les graines qui se sont le plus développées (le soja avec une progression de 17 millions d'hectares et de 55 millions de tonnes, et dans une moindre mesure de colza) et les pays qui ont connu les progressions les plus fortes (essentiellement les États-Unis, le Brésil et l'Argentine) sont des produits et des pays qui ont traditionnellement des rendements moyens nettement supérieurs à la moyenne mondiale. Ce rendement moyen mondial est ainsi passé de $1,3 \mathrm{t} / \mathrm{ha}$ à la fin des années 80 à près de $1,5 \mathrm{t} / \mathrm{ha}$. Ainsi, l'effet surface explique environ 55 \% de la croissance de la production et l'effet rendement $45 \%$.

Durant cette période, outre les États-Unis qui ont vu leur surface en oléagineux s'envoler de plus de 8 millions d'hectares (figure 11), soit deux fois plus que ce qu'ils avaient réalisé entre 1972 et 1990), ce sont l'Inde (+ $6 \mathrm{M}$ d'ha), le Canada (+ $4 \mathrm{M}$ d'ha), l'Argentine (+ $3 \mathrm{M}$ d'ha) et le Brésil (+ $2 \mathrm{M}$ d'ha) qui se sont réparti l'essentiel de l'augmentation des surfaces. La Chine bien que restant le second producteur mondial, n'a vu ses surfaces en oléagineux progresser que de $1 \mathrm{M}$ d'ha. Les progressions de surfaces se sont accompagnées de fortes augmentations des productions de ces mêmes pays, avec naturellement une forte domination des États-Unis (qui ont assuré à eux seuls une augmentation de $28 \mathrm{Mt}$ ), suivi de l'ensemble des deux grands exportateurs d'Amérique du Sud (Argentine et Brésil) avec une progression de $23 \mathrm{Mt}$ et de l'Inde (+ $7 \mathrm{Mt}$ ).

Le commerce mondial des graines oléagineuses a pour sa part augmenté de $20 \mathrm{Mt}$, soit une progression de plus de $60 \%$, sans précédent sur une seule décennie. Aujourd'hui, plus de $18 \%$ de la production mondiale d'oléagineux fait l'objet d'un commerce international contre moins de $15 \%$ il y a dix ans. La tendance que l'on observait historiquement, à savoir le décalage croissant entre les zones mondiales de production de graines et celles de transformation et/ou d'utilisation, n'a pas cessé même si ses moteurs ont changé. Sur la progression de $20 \mathrm{Mt}$ des importations, l'UE à 15 n'a représenté que 5 millions de tonnes (soit une progression certes non négligeable de $33 \%$ ), mais elle est à comparer à l'explosion de la demande chinoise qui, presque inexistante jusqu'au milieu des années 90, a atteint selon Oil World 6,4 Mt en 1998/1999 et plus de 13 Mt en 1999/2000, suite à son 
changement de stratégie qui consiste à privilégier les importations de graines (surtout soja mais aussi à l'occasion colza) pour une transformation locale, en complément, voire à la place des importations directes de tourteaux et d'huiles. Pour des raisons différentes, le Mexique, dont les importations de graines depuis son adhésion à l'Association de libre-échange du Nord de I'Amérique (Alena) sont passées de 1,3 à 5,4 Mt en 1999/2000, constitue aussi un exemple de cette stratégie de transformation locale à partir de graines des autres pays de l'Alena.

Enfin, on doit remarquer que, en dehors du Japon qui a un tonnage relativement stable de 7 à 7,5 Mt, les importations des autres pays du monde ont progressé de plus de $60 \%$ pour dépasser les 15 Mt. De nombreux pays tendent en effet à mettre en place ou à développer leurs activités de trituration (Turquie, Taïwan, Corée, Philippines, Afrique du Nord, etc.). Cette orientation dépend fortement des conditions économiques et géographiques du pays, des capacités d'investissement des acteurs locaux ou des choix des grandes firmes internationales. L'UE, tout en conservant sa place considérable pour les grands exportateurs, n'a plus le même rôle stratégique qu'auparavant. C'est un très gros marché mais avec des perspectives de croissance relativement limitées et de plus en plus exigeant en termes de qualité.

En dix ans, le commerce mondial des matières riches en protéines a progressé de $14 \mathrm{Mt}$, soit $40 \%$, ce qui correspond à un rythme de croissance un peu plus faible que pour les graines. Sur ce total, le tourteau de soja a représenté à lui seul $12 \mathrm{Mt}$. Alors que I'UE a vu son tonnage d'importations augmenter modérément (+ $4 \mathrm{Mt}$ ), ramenant sa part du marché mondial de 59 à $51 \%$, c'est globalement le reste du monde, avec pour une large part les pays d'Asie et d'Amérique du Sud en cours d'industrialisation, qui a connu la plus forte progression avec quasiment un doublement du tonnage, en raison notamment du fort développement de la production de volailles. À l'inverse, les pays de l'ex-URSS s'inscrivent en très fort recul, en raison à la fois de l'effondrement de leur production de viande et de leurs difficultés à financer leurs achats extérieurs.

Pour les corps gras, durant la même période, le commerce a augmenté de $13 \mathrm{Mt}$ (soit $+56 \%$ ) essentiellement sous forme de palme et de soja. L'UE et, dans une moindre mesure, les États-Unis restent des importateurs notables (essentiellement en huiles tropicales) et leur tonnage a augmenté pour chacun de 0,8 Mt. Cependant, le véritable moteur du marché mondial est l'explosion de la demande dans de nombreux pays d'Asie et d'Afrique. L'Inde, I'Afrique du Nord, le Pakistan, I'Iran, etc., ont été les fers de lance de cette croissance. En fait, au niveau mondial, la " consommation " (en fait l'utilisation totale alimentaire et non alimentaire) est passée en moyenne par tête et par an de 15,3 à $18,3 \mathrm{~kg}$ (soit une progression de $20 \%$ ). Dans le même temps, la population mondiale (tout particulièrement dans les pays à faible revenu comme I'Inde, le Pakistan, I'Afrique du Nord et subsaha-rienne) est passée de 5,3 à 6,1 milliards d'habitants (+ 800 millions d'habitants, soit + $15 \%$ ). Les deux phénomènes de croissance démographique et d'augmentation des revenus se sont cumulés. Rien que pour maintenir le niveau moyen actuel de consommation, compte tenu d'un accroissement annuel de population de 80 millions de personnes, il faut 1,5 million de tonnes de corps gras en plus chaque année. De plus, certaines des zones à forte croissance de la demande (comme l'Afrique du Nord, le Moyen-Orient, la Chine, etc.) ont des capacités de développement de la production locale très limitées. 
Notes:

${ }^{1}$ Texte de la conférence prononcée dans le cadre de la Journée de printemps de l'AFECG, placée sous le thème des « Enjeux européens de la filière oléagineuse » (Paris 22 mars 2001).

${ }^{2}$ Elle importe aussi des quantités importantes d'autres matières riches en protéines sous forme de corn-gluten-feed, de farines de poisson, etc.

${ }^{3}$ Le soja est de loin la première graine commercialisée dans le monde avec $48 \mathrm{Mt}$, soit $77 \%$ du total des graines oléagineuses.

${ }^{4}$ Ce tourteau représente les deux tiers de la consommation de I'UE contre près de $90 \%$ de celle des États-Unis.

${ }^{5}$ En fait ces différents paramètres sont $a$ posteriori reliés entre eux, lorsque les ajustements de prix ont eu lieu pour rétablir les différents équilibres au sein de la filière.

${ }^{6}$ Ces QMG se sont traduites par de fortes diminutions des prix d'intervention des différentes graines (colza, tournesol, soja) durant toutes les années où il y a eu dépassement des seuils communautaires ainsi fixés.

${ }^{7}$ Ces SMG ont entraîné de fortes diminutions des aides directes aux oléagineux durant les années de dépassement au niveau communautaire. Cela a affecté les différents pays eux-mêmes en dépassement en 1994/1995, 1996/1997 et 1998/1999.

${ }^{8}$ En particulier, la disparition des aides versées aux triturateurs pour compenser les écarts entre prix communautaires et prix mondiaux des graines de colza et de tournesol.

\section{CONCLUSION}

Dans ce contexte où l'UE n'est plus le débouché essentiel des États-Unis, qui continuent par ailleurs à exercer sur le marché mondial des oléagineux un fort contrôle à travers leur propre politique agricole et les stratégies de leurs grandes firmes de trituration et de négoce, elle se doit d'assurer sa sécurité d'approvisionnement pour le moyen et le long terme et, pour cela, de maintenir la diversité et la spécificité de ses filières oléagineuses et protéagineuses face au "rouleau compresseur " que constitue le soja à l'échelle mondiale. Cela va dans le sens de l'attente des consommateurs européens qui sont de plus en plus sensibles à la qualité, à la traçabilité et à la diversité, aussi bien en termes de goût que de techniques de production, des produits alimentaires. L'UE a connu une longue période de forte croissance quantitative de sa demande intérieure en huile et surtout en tourteaux. Aujourd'hui les perspectives de progression - hors nouveaux secteurs comme le non-alimentaire sont beaucoup plus limitées et le problème se pose davantage en termes qualitatifs. À ce titre I'Europe n'a aucune raison de se laisser imposer des choix qui seraient faits outre-Atlantique. Pour conserver un pouvoir de négociation et d'influence, elle doit donc disposer d'une force de dissuasion par rapport aux marchés internationaux sous forme d'une production locale d'oléagineux et de protéagineux, ainsi que de tourteaux et d'huiles, significative à l'échelle mondiale. 
Pour I'UE, une filière oléo-protéagineuse confortée apparaît comme un " bien public " pour l'ensemble de la collectivité : agriculteurs, transformateurs, consommateurs, citoyens, que le marché n'est pas capable aujourd'hui, à lui seul, de conforter. C'est donc à la fois aux pouvoirs publics, à la recherche et aux différents acteurs économiques qu'il appartient de soutenir spécifiquement ce secteur, au moins jusqu'à ce que de nouvelles conditions de l'environnement international ou de nouveaux mécanismes de marché permettent son fonctionnement autonome.

\section{Illustrations}

\section{UNION EUROPEENNE 15}

\section{ETATS-UNIS}

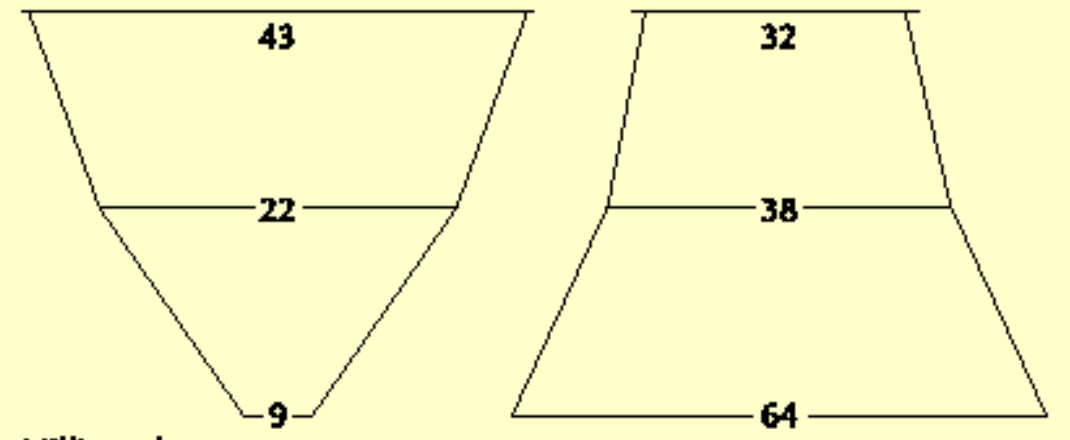

\section{Millions de tonnes}

Figure 1. Comparaison des diagrammes des oléagineux européen et nordaméricain.
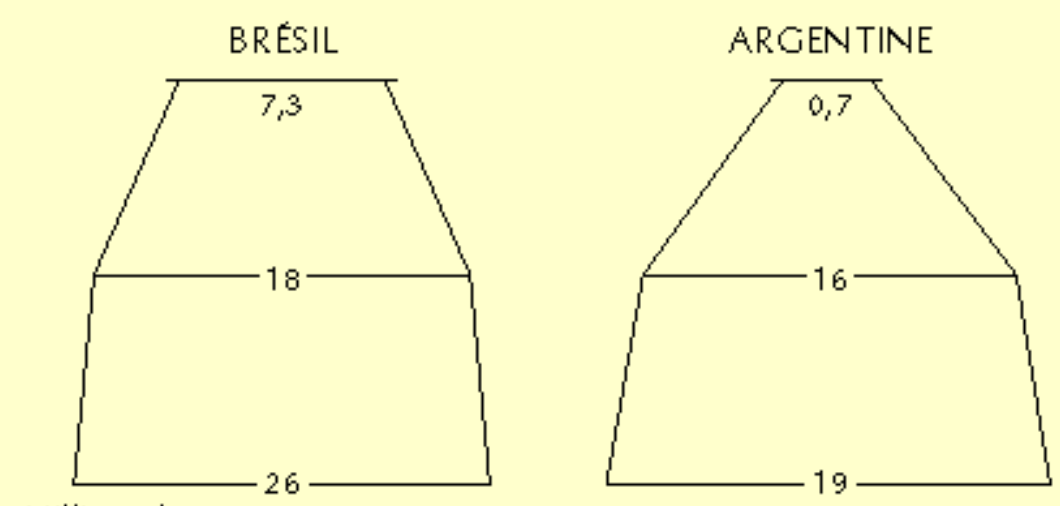

Willions de tonnes

Figure 2. Comparaison des diagrammes des oléagineux brésilien et argentin. 


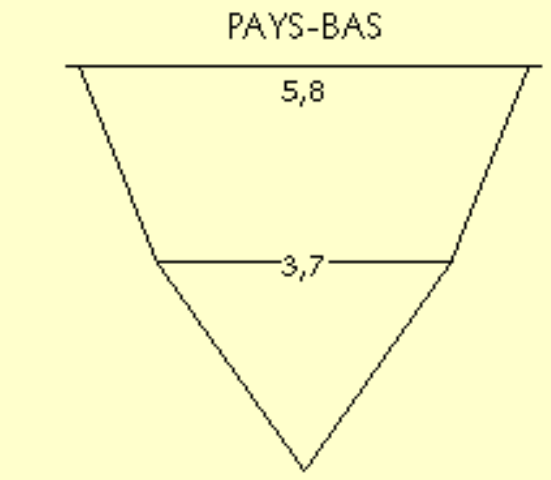

Willions de tonnes

\section{FRANCE}

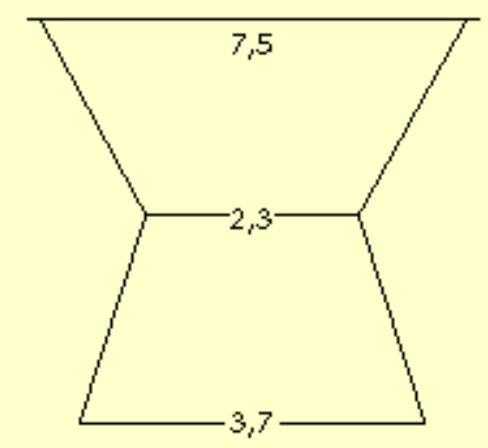

Figure 3. Comparaison des diagrammes des oléagineux néerlandais et français.

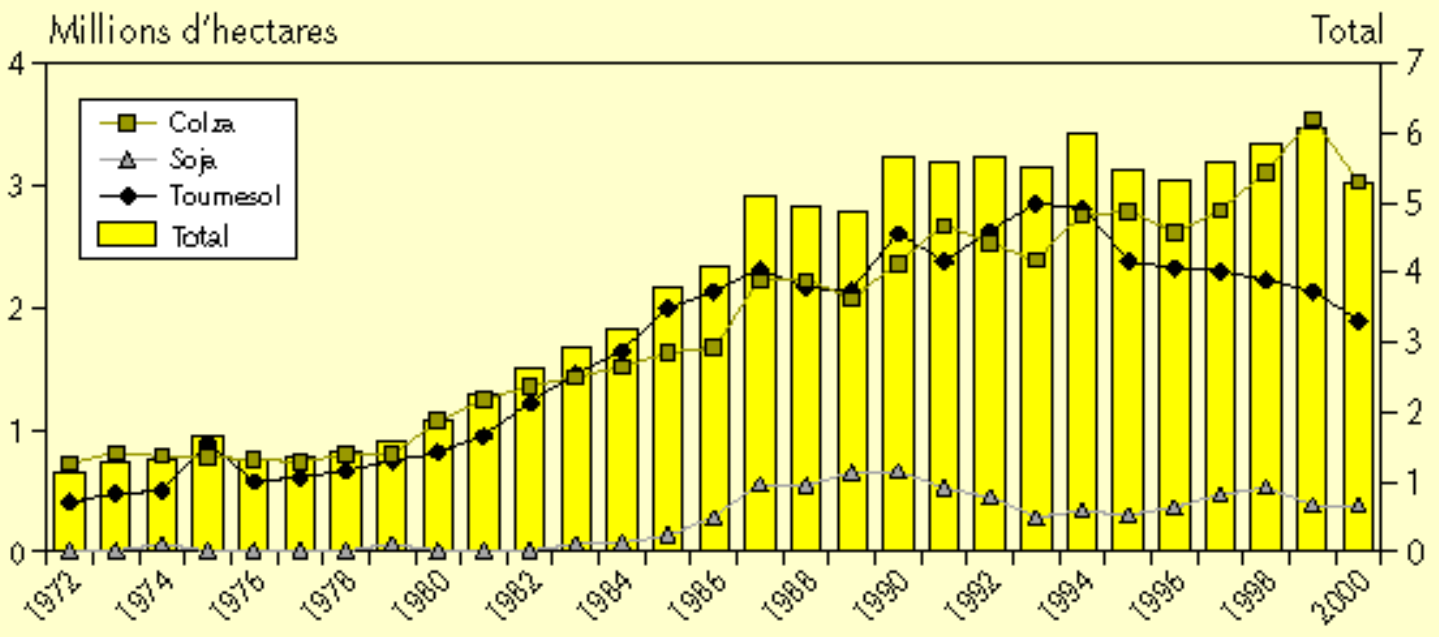

Figure 4. Évolution des surfaces en oléagineux dans I'UE 15. 


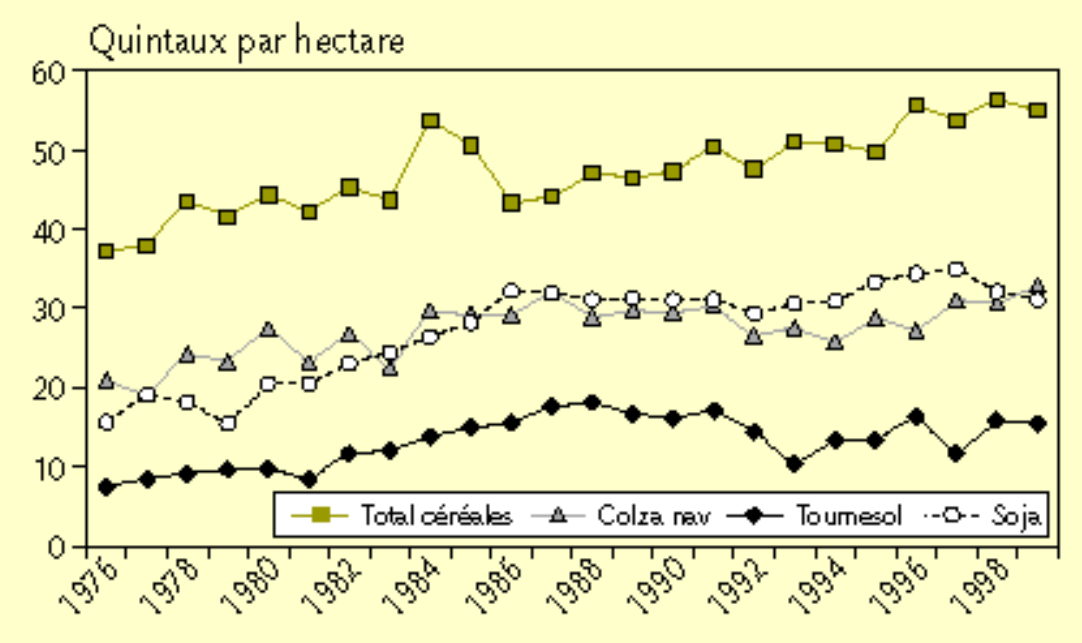

Figure 5. Évolution des rendements dans I'UE.

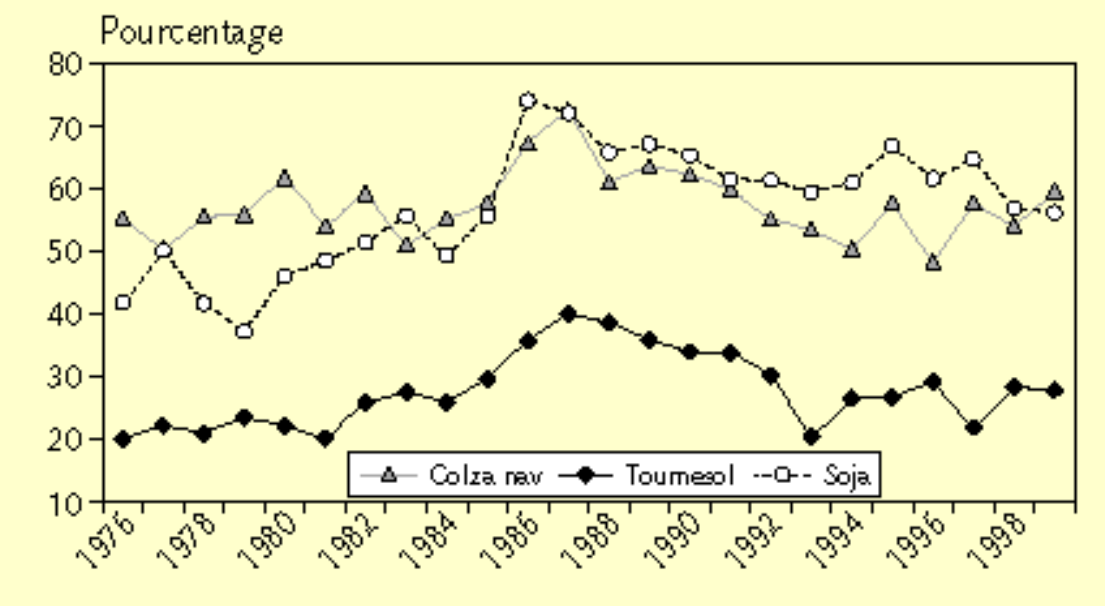

Figure 6. Évolution des rendements relatifs dans I'UE (référence : total céréales). 


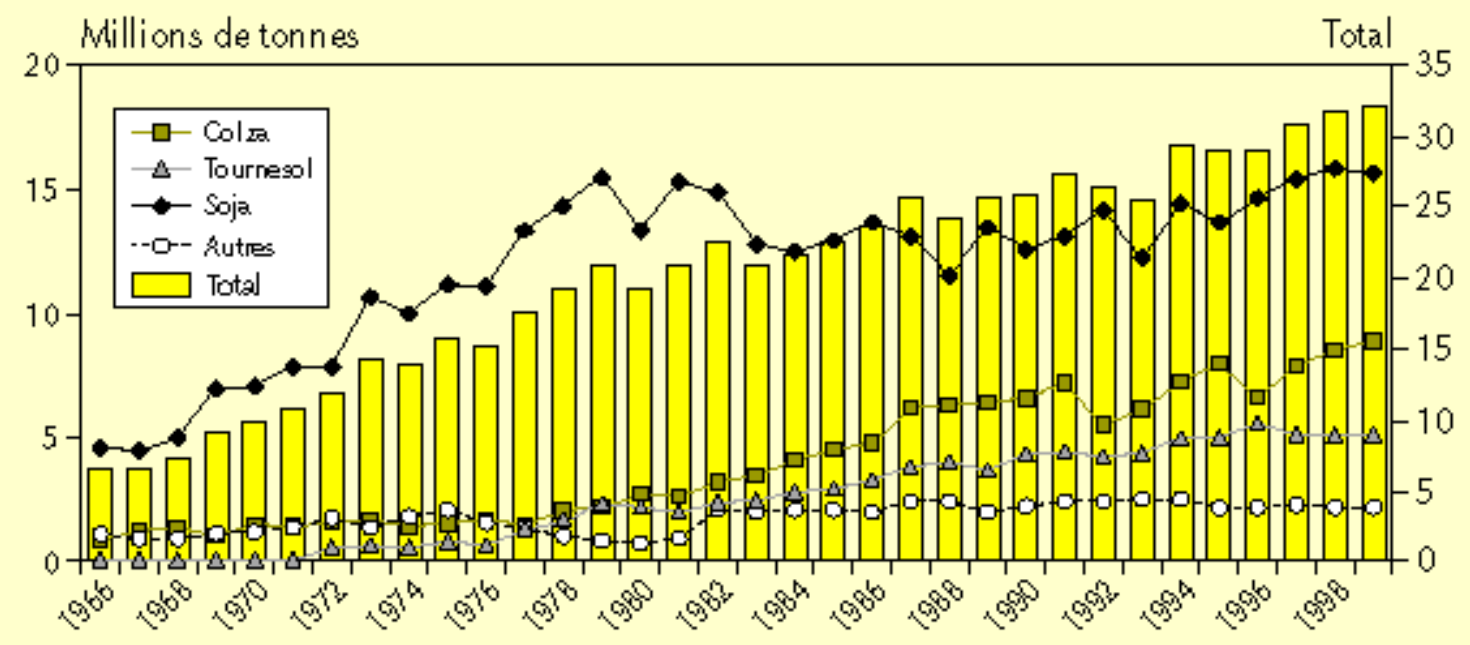

Figure 7. Évolution des triturations de graines dans I'UE 15.

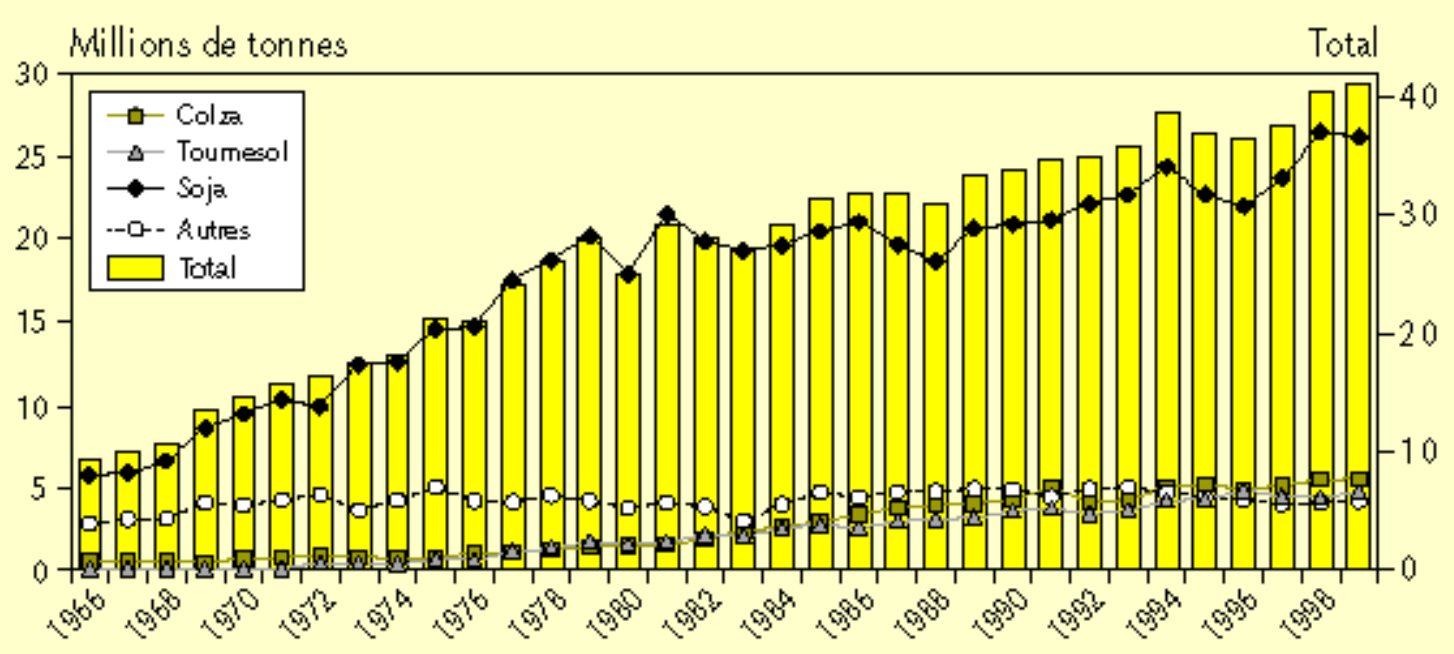

Figure 8. Évolution des consommations de tourteaux, dans I'UE 15. 


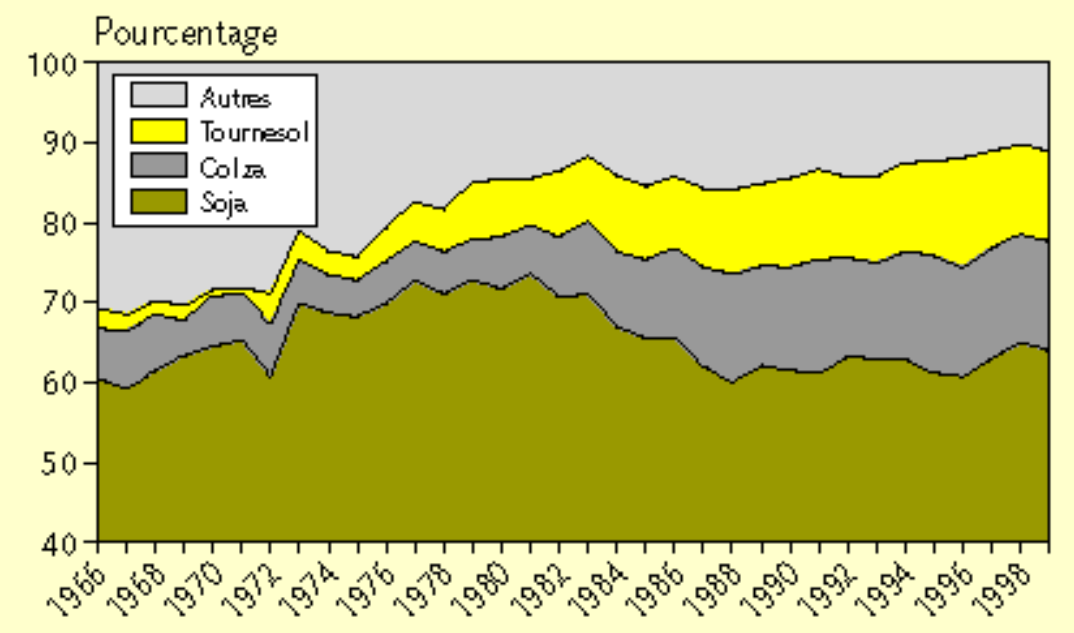

Figure 9. Évolution de la répartition des tourteaux dans I'UE 15.

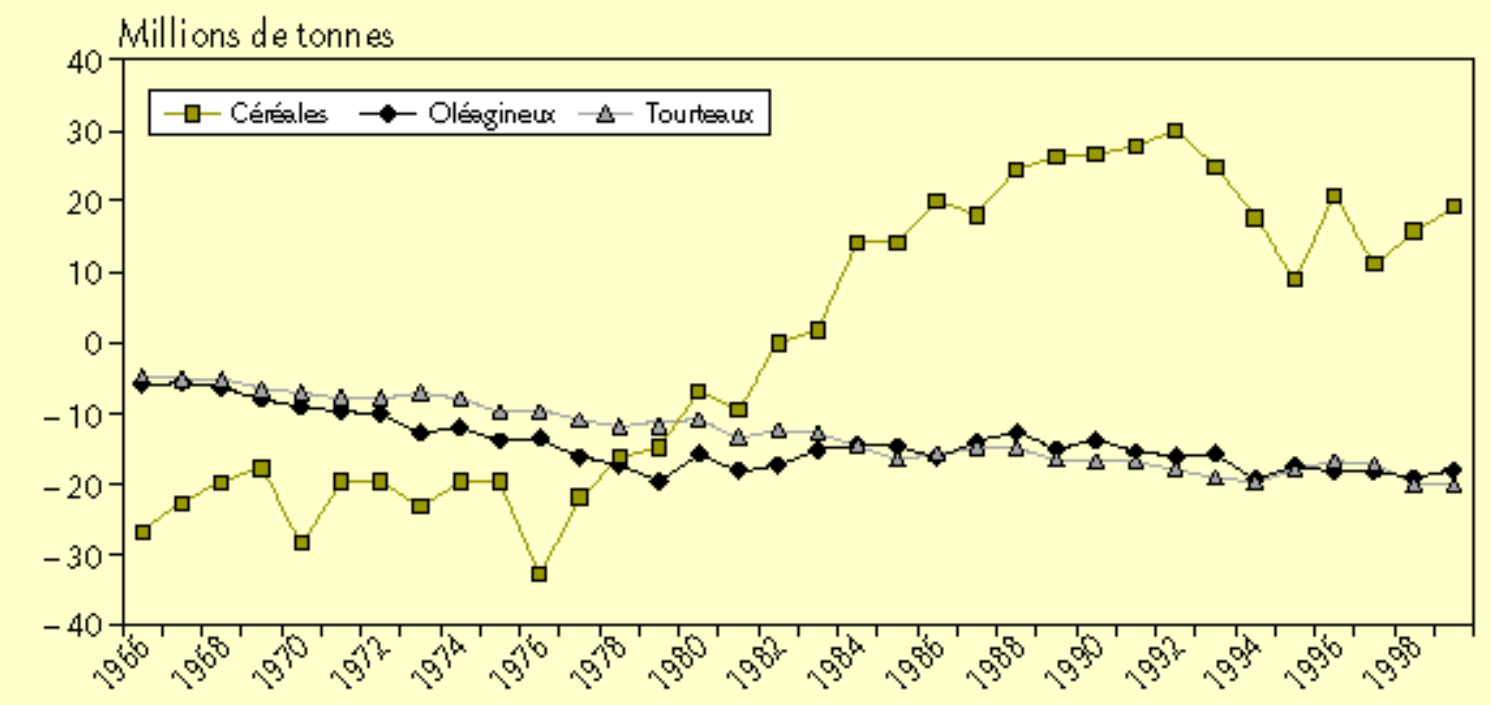

Figure 10. Évolution des soldes dans I'UE 15. 


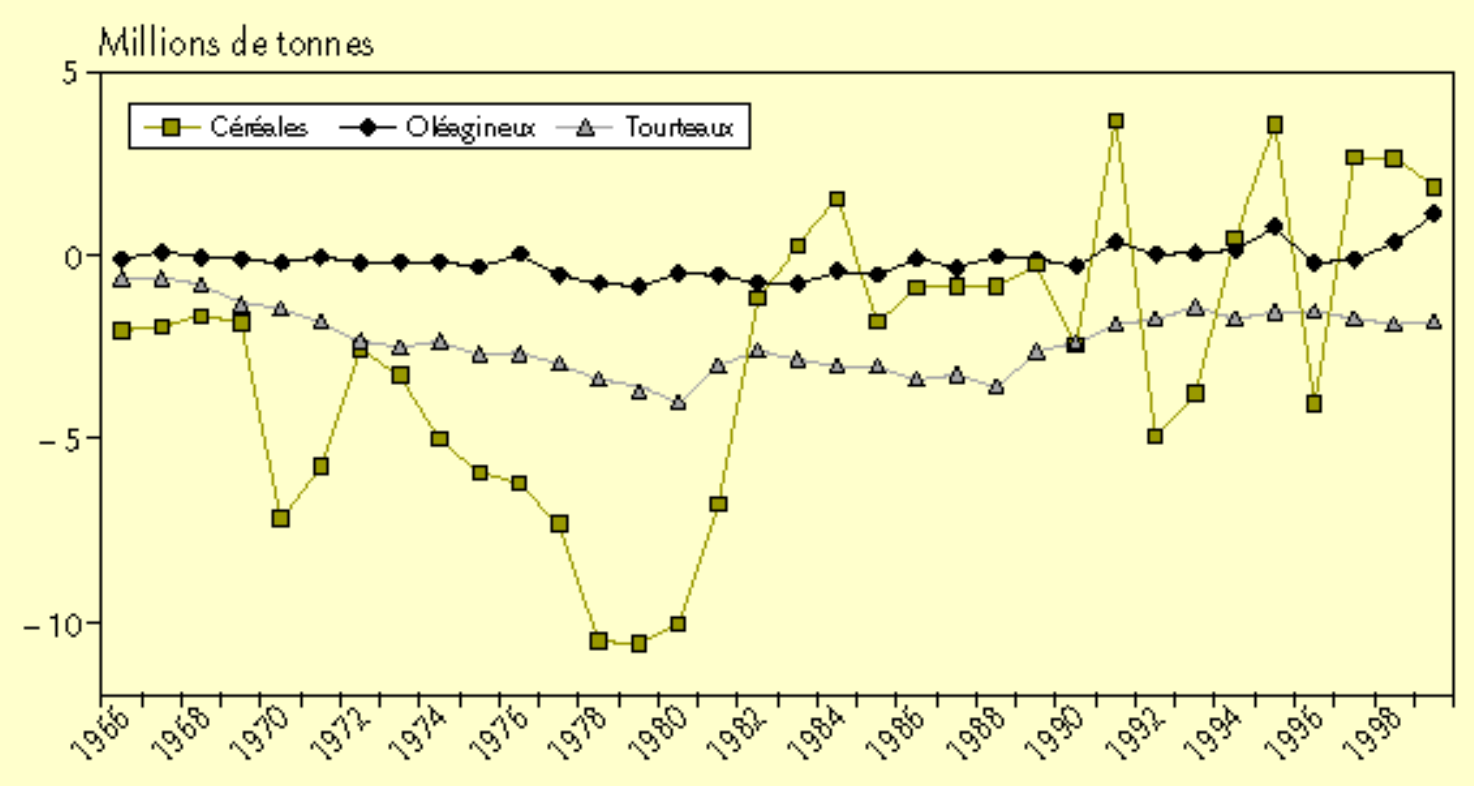

Figure 11. Évolution des soldes des PECOS.

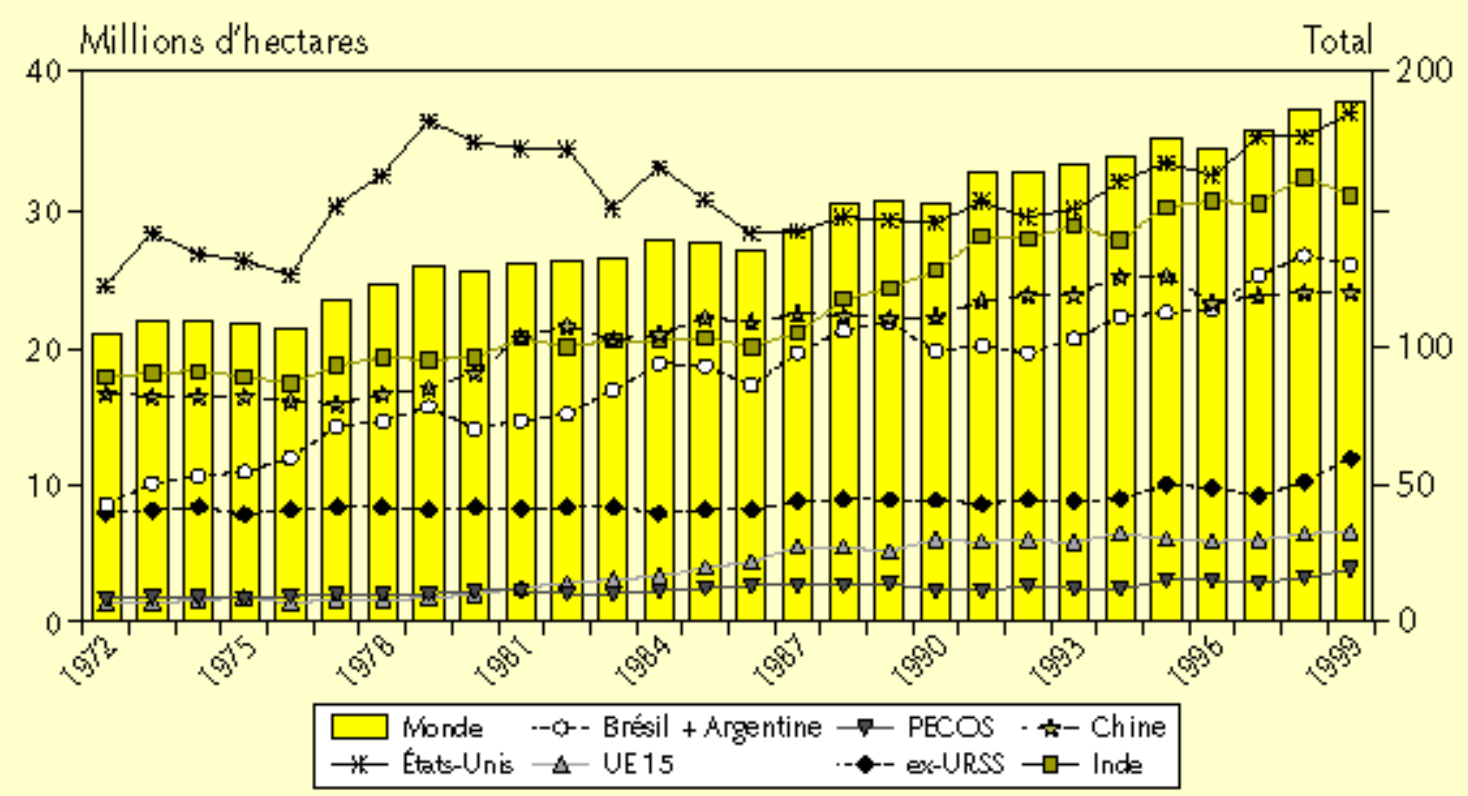

Figure 12. Évolution des surfaces en oléagineux. 
Tableau. Commerce mondial des graines et tourteaux de soja.

\begin{tabular}{|llcccc}
\hline \multirow{4}{*}{ Graines } & États-Unis & Brésil & Argentine & Monde \\
\hline \multirow{6}{*}{ Tourteau } & UE & 6,1 & 7,4 & 0,3 & 15,7 \\
& Chine & 5,2 & 2,1 & 3,1 & 10,4 \\
& Monde & 27,2 & 11,5 & 4,1 & 46,8 \\
& UE & 0,5 & 7,6 & 7,9 & 17,1 \\
& Chine & 0 & 0,1 & 0,3 & 0,5 \\
& Monde & 7,1 & 9,6 & 13,2 & 50,1
\end{tabular}

Un ités : million s de tonnes. 